\title{
Birth Cohort Testing for Hepatitis C Virus — Indian Health Service 2012-2015
}

\author{
Brigg Reilley, $\mathrm{MPH}^{1}$; Jessica Leston, $\mathrm{MPH}^{2}$; Susan Hariri, $\mathrm{PhD}^{3}$; Lisa Neel, $\mathrm{MPH}^{1}$; Miles Rudd, $\mathrm{MD}^{4}$; \\ Megan Galope, $\mathrm{MBA}^{5}$; John Ward, $\mathrm{MD}^{3}$; Claudia Vellozzi, $\mathrm{MD}^{3}$
}

Hepatitis $\mathrm{C}$ virus (HCV) infection is a substantial and largely unrecognized public health problem. An estimated 3.5 million persons in the United States are currently living with HCV infection, at least half of whom are unaware of their infection (1-3). Persons born during 1945-1965 (the "baby boomer" birth cohort) have a sixfold higher prevalence $(2.6 \%)$ than adults of other ages, and represent $81 \%$ of all persons chronically infected with $\mathrm{HCV}(4)$. Therefore, in addition to recommending testing for all persons at risk for $\mathrm{HCV}$ infection, CDC and the U.S. Preventive Services Task Force (USPSTF) recommend one-time HCV testing for the birth cohort $(5,6)$. Compared with the national average, American Indian/Alaska Native (AI/AN) persons have approximately twofold the rate of acute $\mathrm{HCV}$ incidence and $\mathrm{HCV}$ associated mortality (2). In June 2012, the Indian Health Service (IHS) implemented HCV testing in the 1945-1965 birth cohort and created a nationally standardized performance measure to monitor implementation of the recommendation. As of June 2015, the proportion of the birth cohort screened for HCV increased from a baseline of $7.9 \%(14,402 / 182,503)$ to $32.5 \%$ $(68,514 / 211,014)$ among the AI/AN population served by IHS nationwide; provider training and the use of clinical decision tools were associated with increases in $\mathrm{HCV}$ testing. With this fourfold increase in testing in just 3 years, IHS needs to prepare for the challenges associated with increased identification of persons living with $\mathrm{HCV}$ infection.

IHS provides care to approximately 1.9 million AI/AN members of 566 federally recognized tribes through a large network of health care facilities. IHS operates 46 hospitals, 344 health centers, and 230 village clinics and health stations in 35 states. $^{*}$ Among hospitals and health clinics, 77\% (300/390) are tribally operated, and the remainder are federally operated. Most facilities provide primary care in remote and rural settings.

An estimated $85 \%$ of IHS facilities use a common electronic health record (EHR), which routinely provides data to monitor a set of preventive health performance measures through the electronic Clinical Reporting System (CRS). In 2011, annual ${ }^{\dagger} \mathrm{HCV}$ antibody testing of the birth cohort was added as a performance measure to establish a baseline in anticipation

\footnotetext{
*An IHS health station is an ambulatory care facility (fixed or mobile) that is geographically separate from an inpatient hospital or health center, provides one or more clinical services, and is operated $<40$ hours per week.

$\dagger$ The IHS CRS data year is July-June. All annual data cited in this report for 2012-2015 represents the data report period of July 2011-June 2015.
}

of the release of expanded CDC recommendations for HCV testing in August 2012. HCV testing coverage is measured as the proportion of the total health care users within the population (i.e., AI/AN residents of a defined catchment community with at least one clinical visit in the past 3 years) born during 1945-1965 with at least one documented HCV antibody test and no previous recorded diagnosis of $\mathrm{HCV}$ infection. Persons with current $\mathrm{HCV}$ infection were identified using International Classification of Disease (ICD) codes. HCV antibody testing was ascertained using Current Procedural Terminology (CPT), Logical Observation Identifiers Names and Codes, or local facility taxonomies. ${ }^{\S}$ Nationally, CRS reports HCV antibody tests performed in patients in all federal and tribal facilities by sex and age. Data from federally operated IHS facilities were further stratified by facility, sex, and age; because of current data sharing agreements, such stratification was not performed on data from tribal facilities. No patient-level data are shared on the CRS platform. Results from HCV antibody tests are not available in aggregate, and thus, are not reported here.

Because IHS facilities are decentralized, implementation of $\mathrm{HCV}$ testing for persons in the birth cohort is a local decision based on capacity and priorities. With the publication of $\mathrm{HCV}$ screening recommendations by CDC in August 2012, support for HCV testing was integrated into existing programs using methods and strategies that have been documented as successful in IHS facilities (e.g., EHR clinical decision support tools, local testing policies, and nursing collaborative agreements to order laboratory tests for indicated testing procedures) (7). Based on best practices identified nationally, regionally, and locally, IHS also implemented clinical trainings and obtained telehealth support. Provider training and other technical assistance were offered to all facilities; however, their use was optional.

During 2012-2015, the unique birth cohort patients tested for HCV antibody in combined federal and tribal IHS facilities increased fourfold from $14,402(7.9 \%)$ to 68,514 (32.5\%) (Table 1). HCV testing was higher among females across all years (Table 1). The 62 federally operated service units accounted for $53 \%(112,319 / 211,014)$ of the total IHS birth cohort population eligible for testing in 2015; facilities in the

\footnotetext{
\$ $\mathrm{HCV}$ purpose of visit codes defined as ICD-9 codes $070.41,070.44,070.51$, 070.54, 070.70 through 070.71, V02.62; ICD-10: B17.10, B17.11, B18.2, B19.20, and B19.21. HCV antibody test is determined by CPT 86803 .

T The 90 federally operated physical health care facilities are grouped into 62 federally operated service units for purposes of administration and data. For example, a service unit might consist of a hospital plus two nearby clinics.
} 
TABLE 1. Hepatitis C virus antibody testing (cumulative) among persons born during 1945-1965, by total eligible population and sex — Indian Health Service, 2012-2015

\begin{tabular}{lrrr}
\hline $\begin{array}{l}\text { Year (total eligible } \\
\text { population) }\end{array}$ & $\begin{array}{c}\text { No. tested } \\
\text { (\% coverage) }\end{array}$ & No. male (\%) & No. female (\%) \\
\hline $2012(\mathrm{~N}=182,503)$ & $14,402(7.9)$ & $5,617(6.8)$ & $8,785(8.7)$ \\
$2013(\mathrm{~N}=195,623)$ & $20,419(10.4)$ & $7,591(8.7)$ & $12,828(11.9)$ \\
$2014(\mathrm{~N}=214,340)$ & $52,971(24.7)$ & $20,859(21.8)$ & $32,112(27.0)$ \\
$2015(\mathrm{~N}=211,014)$ & $68,514(32.5)$ & $27,636(29.3)$ & $40,878(35.0)$ \\
\hline
\end{tabular}

Southwest region, which is the most populous among the federally operated facilities, had the highest testing rates (Table 2).

By June 2015, the proportion of the birth cohort tested by geographic regions varied from $31.2 \%$ to $41.2 \%$. However, there was much greater variation in birth cohort testing observed by facility, ranging from $1.9 \%$ to $75.1 \%$. The average testing coverage of the birth cohort among federal facilities in the top testing quartile was $58.5 \%(17,288 / 29,606) ; 14$ (93\%) of the 15 facilities had deployed the HCV clinical decision support testing reminder as of June 2015. Among the bottom quartile of federal facilities, none had implemented a clinical decision tool; average testing coverage of the birth cohort was $16.4 \%(2,781 / 17,128)$.

\section{Discussion}

IHS expansion of HCV testing of the birth cohort has been highly successful, resulting in a fourfold increase in testing overall. Substantial increases were observed nationwide, with wide variability at the facility level. Testing rates in all regions were higher for women, possibly attributable to higher rates of health care utilization and therefore more opportunities to screen.

Reductions in HCV-associated morbidity and mortality are only possible through follow-up of persons found to be seropositive, including confirmation of HCV viremia, genotyping, and liver disease staging/assessment for treatment. To increase the likelihood of successful treatment outcomes, it is also critical that persons living with $\mathrm{HCV}$ receive counseling and are linked to behavioral health interventions, including those aimed at reducing alcohol use when appropriate. Compared with other racial/ethnic populations, alcohol-related death rates are higher among AI/ANs, placing these persons at increased risk for progression of $\mathrm{HCV}$-associated morbidity and liver disease (8). Other cofactors affecting disease progression (e.g., human immunodeficiency virus) must also be considered as part of routine clinical follow-up care for HCV infection.

Follow-up HCV care and treatment services for persons tested and found to be living with HCV infection have been implemented in several IHS facilities, including some that are located in remote settings with limited referral options. These care and treatment programs are led by a variety of primary
TABLE 2. Hepatitis $C$ virus antibody testing (cumulative) among persons born during 1945-1965, by Indian Health Service federally operated facilities and region, ${ }^{*} 2015$

\begin{tabular}{lccrr}
\hline Region & $\begin{array}{c}\text { No. of } \\
\text { facilities }\end{array}$ & $\begin{array}{c}\text { Total } \\
\text { eligible } \\
\text { population }\end{array}$ & $\begin{array}{c}\text { No. tested } \\
\text { (\% coverage) }\end{array}$ & $\begin{array}{c}\text { Coverage } \\
\text { range among } \\
\text { facilities }\end{array}$ \\
\hline Northern plains & 21 & 31,206 & $9,927(31.8)$ & $18.4 \%-66.6 \%$ \\
Southern plains & 12 & 9,579 & $3,009(31.4)$ & $6.5 \%-70.5 \%$ \\
Southwest & 21 & 64,120 & $26,424(41.2)$ & $1.9 \%-69.4 \%$ \\
East & 3 & 602 & $188(31.2)$ & $15.6 \%-44.7 \%$ \\
Pacific coast & 6 & 6,812 & $2,444(35.9)$ & $21.3 \%-75.1 \%$ \\
Total & 62 & 112,319 & $41,992(37.4)$ & $1.9 \%-75.1 \%$ \\
\hline
\end{tabular}

* Northern plains: lowa, Minnesota, Montana, Nebraska, South Dakota; Southern plains: Oklahoma, Texas; Southwest: Arizona, Colorado, New Mexico, Nevada, Utah; East: Maine, Massachusetts, North Carolina; Pacific coast: Idaho, Oregon, Washington.

care providers (e.g., physicians, nurses, and pharmacists), generally in collaboration with a specialist through telehealth programs that have proven to be effective (e.g., Extension for Community Healthcare Outreach programs) (9).

Although IHS has had success in increasing HCV testing, challenges remain. Clinical capacity remains a substantial barrier to providing the care and treatment necessary for cure. Despite the availability of highly effective and safe HCV therapies that can reach sustained virologic response (cure) within 12 weeks, some primary care providers remain hesitant to provide treatment because they associate $\mathrm{HCV}$ medications with previously used interferon-based treatment regimens that were complicated, lengthy, and poorly tolerated. Furthermore, the cost of the new HCV drugs can be prohibitive; currently, the vast majority of IHS patients obtain $\mathrm{HCV}$ medication at no cost through state Medicaid and pharmaceutical manufacturer patient assistance programs. It is unclear if this approach will be sustainable as a larger number of AI/AN persons with HCV infection are identified and linked to care.

The findings in this report are subject to at least four limitations. First, HCV seroprevalence could not be estimated at a national level due to lack of laboratory test results or other standardized and reliable indicators of infection. However, work is underway to identify and standardize data sources to obtain accurate national estimates; preliminary data suggest estimates of $\mathrm{HCV}$ antibody or RNA positivity range from $2 \%$ to $10 \%$ based on ICD codes. Second, migration of approximately $15 \%$ of facilities to private sector electronic health records that do not interface with IHS electronic systems are not included in this analysis. Third, improvements in HCV testing cannot be attributed to any specific intervention strategies using observational data. However, the higher rates of HCV testing observed among the facilities that implemented an EHR decision tool compared with those that did not suggest that such tools substantially contributed to higher testing rates. Finally, provider training and other technical assistance 


\section{Summary}

What is already known about this topic?

Hepatitis C virus (HCV) is an increasing cause of morbidity and mortality in the United States, and disproportionally affects American Indians/Alaska Natives. Curative HCV therapies provide an opportunity to reduce the prevalence of HCV infection in the United States. Adults born during 1945-1965 (birth cohort) account for approximately $75 \%$ of the estimated 3.5 million persons with HCV infections in the United States and are recommended to receive one-time testing for HCV.

What is added by this report?

In June 2012, the Indian Health Service (IHS) implemented national recommendations for one-time HCV testing in the birth cohort. During 2012-2015 HCV testing coverage in the American Indian/Alaska Native birth cohort increased from $7.9 \%$ to $32.5 \%$ in IHS facilities serving largely remote and rural populations across 35 states. Testing coverage in individual IHS facilities ranged from $1.9 \%$ to $75.1 \%$; the largest increase occurred among facilities that deployed an electronic clinical decision support tool for HCV testing.

What are the implications for public health practice?

Identifying persons with HCV infection in American Indian/ Alaska Native populations is a priority. Implementation of clinical decision tools should be considered to improve testing and detection; clinical capacity should be adequate to provide the follow-up care and treatment necessary for cure.

could not be quantified systematically, because response and implementation varied widely among IHS facilities. Additional investigation is needed to better understand why some facilities have adopted testing more readily than others and to determine the extent to which use of clinical decision tool reminders contributed to higher coverage rates.

HCV-related morbidity and mortality are now largely preventable, and IHS is committed to ensuring that HCV infections are diagnosed, and that persons with $\mathrm{HCV}$ infection in AI/AN communities receive timely access to care and treatment. Successful implementation of HCV testing of the birth cohort as recommended by CDC and USPSTF has been demonstrated in IHS facilities providing health care services in largely remote and rural sites and might represent best practices for health networks operating in similar settings. Next steps include estimating seroprevalence of and confirmation of HCV infection in preparation to scale up the capacity to care for AI/AN living with current infection. IHS will also continue to develop strategies to overcome anticipated future cost-associated barriers to HCV treatment and cure.

\section{Acknowledgments}

James Gemelas, Patrice Roberts, Jonathan Iralu, California Area Indian Health Service Government Performance and Results Act office; Diane Leach, James Spillane, Mose Herne.

\footnotetext{
${ }^{1}$ National HIV/AIDS Program, Office of Clinical and Preventive Services, Indian Health Service (IHS), Maryland; ${ }^{2}$ Northwest Portland Area Indian Health Board, Oregon; ${ }^{3}$ Division of Viral Hepatitis, National Center for HIV/ AIDS, Viral Hepatitis, STD, and TB Prevention, CDC; ${ }^{4}$ Portland Area Office, IHS, Oregon; ${ }^{5}$ Office of Information Technology, IHS.
}

Corresponding author: Brigg Reilley, brigg.reilley@ihs.gov, 646-932-6493.

\section{References}

1. Edlin BR, Eckhardt BJ, Shu MA, Holmberg SD, Swan T. Toward a more accurate estimate of the prevalence of hepatitis $\mathrm{C}$ in the United States. Hepatology 2015;62:1353-63. http://dx.doi.org/10.1002/hep.27978

2. CDC. Viral hepatitis surveillance, United States. Atlanta, GA: US Department of Health and Human Services, CDC; 2013. http://www.cdc. gov/hepatitis/statistics/2013surveillance/pdfs/2013hepsurveillancerpt.pdf

3. Byrd KK, Redd JT, Holman RC, Haberling DL, Cheek JE. Changing trends in viral hepatitis-associated hospitalizations in the American Indian/ Alaska Native population, 1995-2007. Public Health Rep 2011;126:816-25.

4. Denniston MM, Jiles RB, Drobeniuc J, et al. Chronic hepatitis C virus infection in the United States, National Health and Nutrition Examination Survey 2003 to 2010. Ann Intern Med 2014;160:293-300. http://dx.doi. org/10.7326/M13-1133

5. Smith BD, Morgan RL, Beckett GA, et al. Recommendations for the identification of chronic hepatitis $\mathrm{C}$ virus infection among persons born during 1945-1965. MMWR Recomm Rep 2012;61(No. RR-4).

6. US Preventive Services Task Force. Final recommendation statement, hepatitis C: screening. Rockville, MD: US Preventive Services Task Force; 2013. http://www.uspreventiveservicestaskforce.org/Page/Document/ UpdateSummaryFinal/hepatitis-c-screening

7. Reilley B, Leston J, Tulloch S, Neel L, Galope M, Taylor M. Implementation of national HIV screening recommendations in the Indian Health Service. J Int Assoc Provid AIDS Care 2015;14:291-4. http://dx.doi.org/10.1177/2325957415570744

8. Landen M, Roeber J, Naimi T, Nielsen L, Sewell M. Alcohol-attributable mortality among American Indians and Alaska Natives in the United States, 1999-2009. Am J Public Health 2014;104(Suppl 3):S343-9. http://dx.doi.org/10.2105/AJPH.2013.301648

9. Arora S, Thornton K, Murata G, et al. Outcomes of treatment for hepatitis $\mathrm{C}$ virus infection by primary care providers. N Engl J Med 2011;364:2199-207. http://dx.doi.org/10.1056/NEJMoa1009370 